\title{
Neutron skins in spherical and deformed nuclei from Skyrme HF
}

CSIC
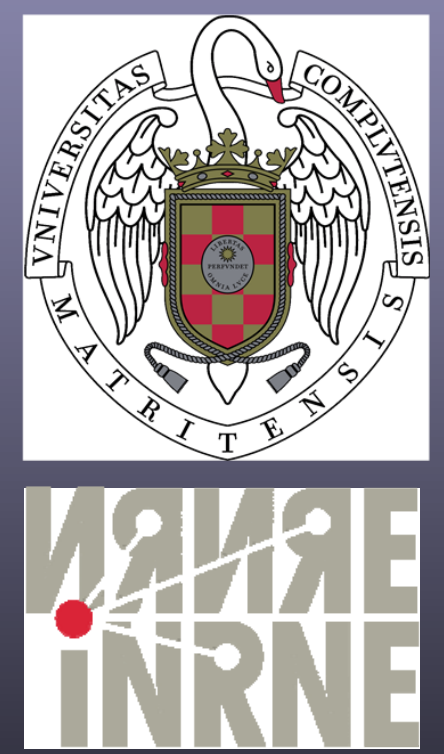

P. Sarriguren (IEM-CSIC, Spain)

E.Moya de Guerra (UCM, Spain)

M.K. Gaidarov (INRN, Bulgaria)

A.N. Antonov (INRN, Bulgaria) 


\section{Outline}

\section{Motivation :}

$>$ New phenomena in nuclei far away from the stability valley

$>$ Neutron-skin formation in n-rich nuclei

$>$ Density dependence of the symmetry energy

$>$ EOS of asymmetric nuclear matter

\section{Theoretical approach :}

$>$ Deformed HF+BCS formalism with Skyrme forces (SLy4)

\section{Results :}

> Proton, neutron and charge rms radii ( $\mathrm{Ni}, \mathrm{Kr}$ and $\mathrm{Sn}$ isotopes)

$>$ Neutron skin thickness from various definitions

$>$ Neutron skin thickness in deformed nuclei 


\section{Skyrme Hartree-Fock}

Schroedinger equation

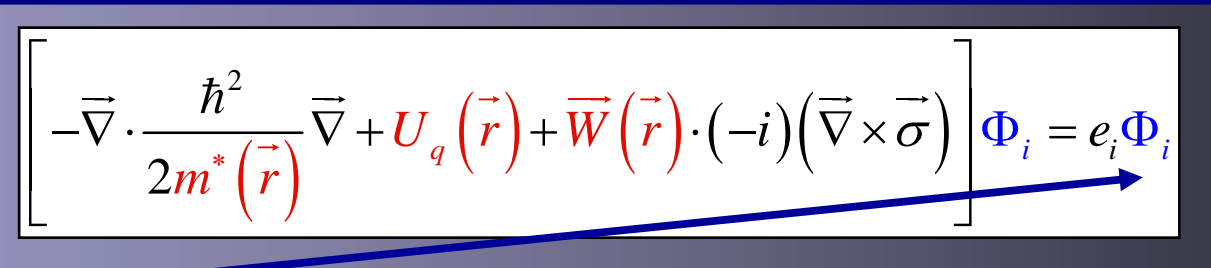

$m^{*}(\vec{r}), \quad U_{q}(\vec{r}), \quad \vec{W}(\vec{r})$

$$
\rho(\vec{r})=\sum_{i}\left|\Phi_{i}(\vec{r})\right|^{2}
$$

Axially deformed Skyrme Hartree-Fock

$$
\Phi_{i}(\vec{R}, \sigma, q)=\chi_{q_{i}}(q)\left[\Phi_{i}^{+}(r, z) e^{i \Lambda^{-} \varphi} \chi_{+1 / 2}(\sigma)+\Phi_{i}^{-}(r, z) e^{i \Lambda^{+} \varphi} \chi_{-1 / 2}(\sigma)\right]
$$

Expansion into eigenfunctions of deformed harmonic oscillator

$$
\begin{array}{r}
\Phi_{i}(\vec{R}, \sigma, q)=\chi_{q_{i}} \sum_{\alpha} C_{\alpha}^{i} \phi_{\alpha}(\vec{R}, \sigma) \quad \alpha=\left\{n_{r}, n_{z}, \Lambda, \Sigma\right\} \\
\rho(r, z)=\sum_{i}\left[\left|\Phi_{i}^{+}(r, z)\right|^{2}+\left|\Phi_{i}^{-}(r, z)\right|^{2}\right]
\end{array}
$$




\section{Pairing correlations in BCS approximation}

\section{Pairing interaction $G$}

$$
H=\sum_{k>0} \epsilon_{k}\left(a_{k}^{+} a_{k}+a_{\bar{k}}^{+} a_{\bar{k}}\right)-G \sum_{k k^{\prime}>0} a_{k}^{+} a_{\bar{k}}^{+} a_{\bar{k}^{\prime}} a_{k^{\prime}}
$$

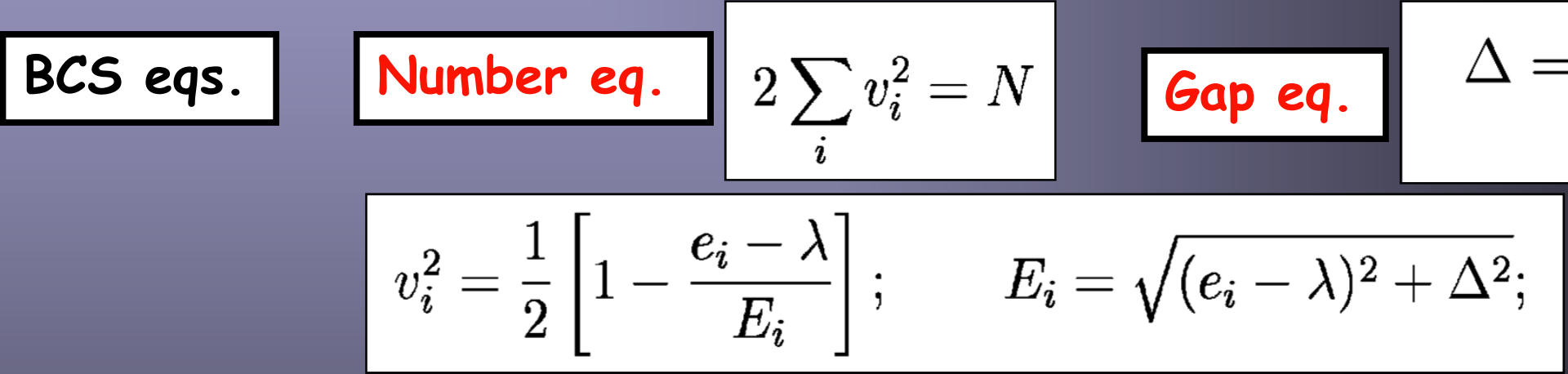$$
\rho(r, z)=\sum_{i} 2 v_{i}^{2}\left[\left|\Phi_{i}^{+}(r, z)\right|^{2}+\left|\Phi_{i}^{-}(r, z)\right|^{2}\right]
$$ 


\section{Densities, radii, and form factors}

\section{Multipole decomposition}

$$
\begin{aligned}
& \rho(r, z)=\sum_{\lambda} \rho_{\lambda}(R) P_{\lambda}(\cos \theta)=\rho_{0}(R)+\rho_{2}(R) P_{2}(\cos \theta)+\cdots \\
& \rho_{\lambda}(R)=\frac{2 \lambda+1}{2} \int_{-1}^{+1} P_{\lambda}(\cos \theta) \rho(R \cos \theta, R \sin \theta) d(\cos \theta)
\end{aligned}
$$

\section{Normalization}

$$
\int \rho(\vec{R}) d \vec{R}=X ; 4 \pi \int \rho_{0}(R) R^{2} d R=X ; \quad X=Z, N
$$

Mean square radii

$$
\left\langle r_{p, n}^{2}\right\rangle=\frac{\int R^{2} \rho_{p, n}(\vec{R}) d \vec{R}}{\int \rho_{p, n}(\vec{R}) d \vec{R}}
$$

Formfactors

$$
F_{p, n}(\vec{q})=\frac{\int \rho_{p, n}(\vec{R}) e^{i \vec{q} \cdot \vec{R}} d \vec{R}}{\int \rho_{p, n}(\vec{R}) d \vec{R}}
$$




\section{Helm model}

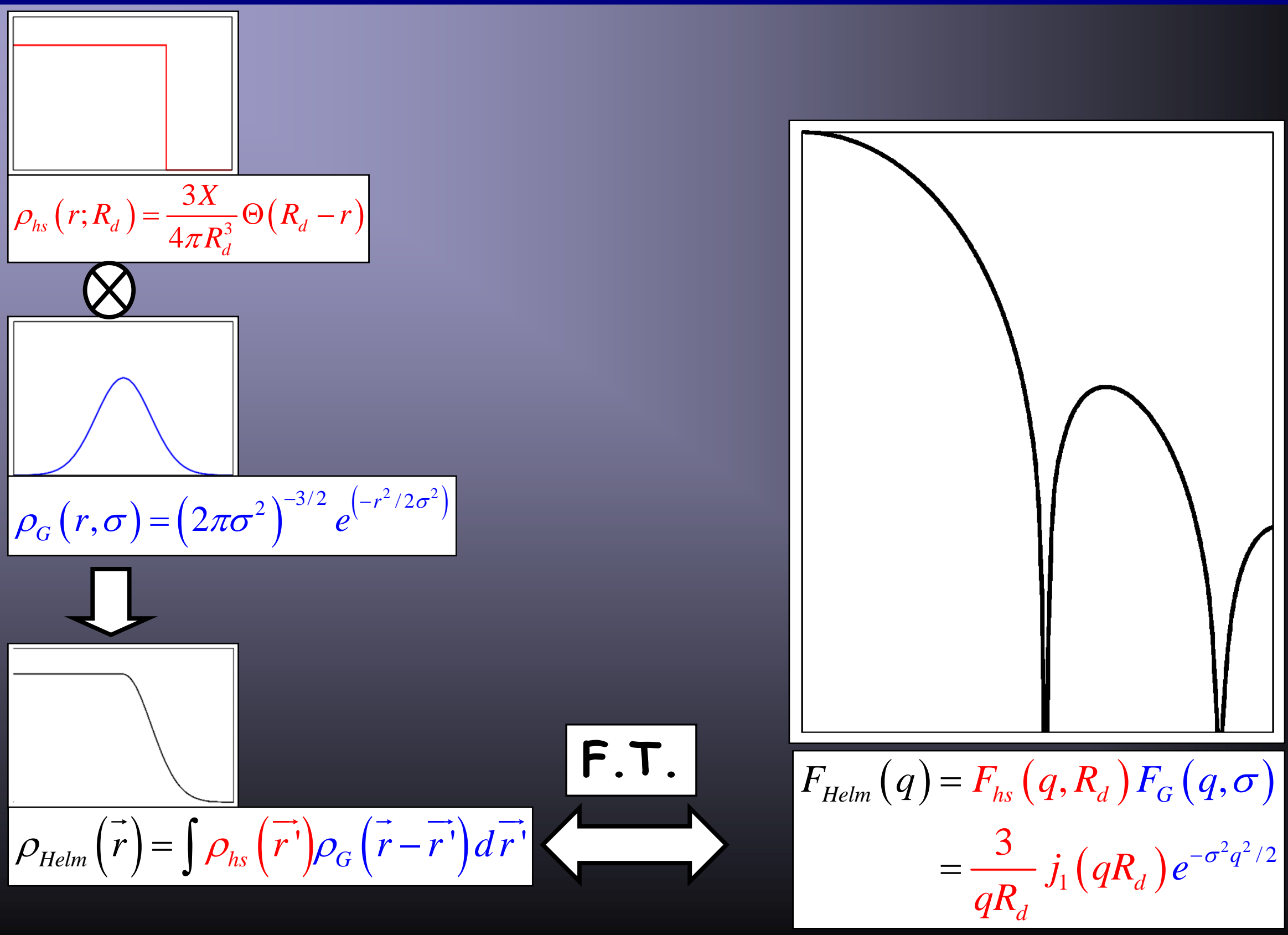




\section{Helm model}

\section{$R_{d}=4.49341 / q_{1}$}

$\rho_{h s}\left(r ; R_{d}\right)=\frac{3 X}{4 \pi R_{d}^{3}} \Theta\left(R_{d}-r\right)$

$\otimes$

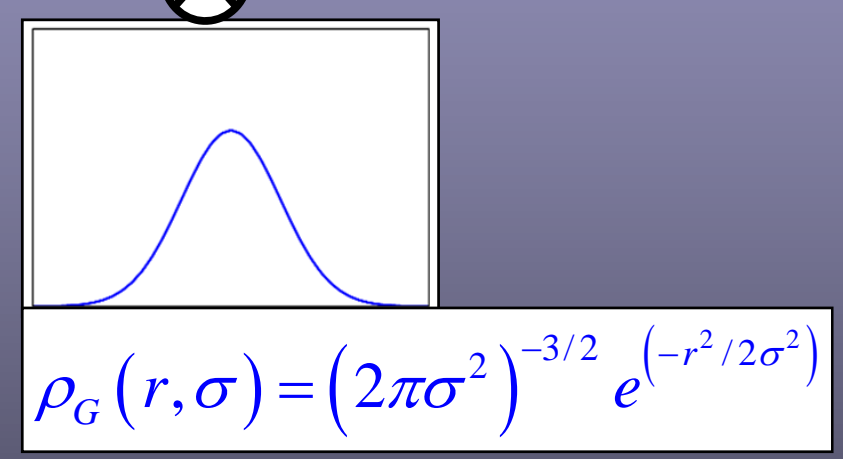

F.T.

$\rho_{\text {Helm }}(\vec{r})=\int \rho_{\text {hs }}\left(\overrightarrow{r^{\prime}}\right) \rho_{G}\left(\vec{r}-\overrightarrow{r^{\prime}}\right) d \overrightarrow{r^{\prime}}$

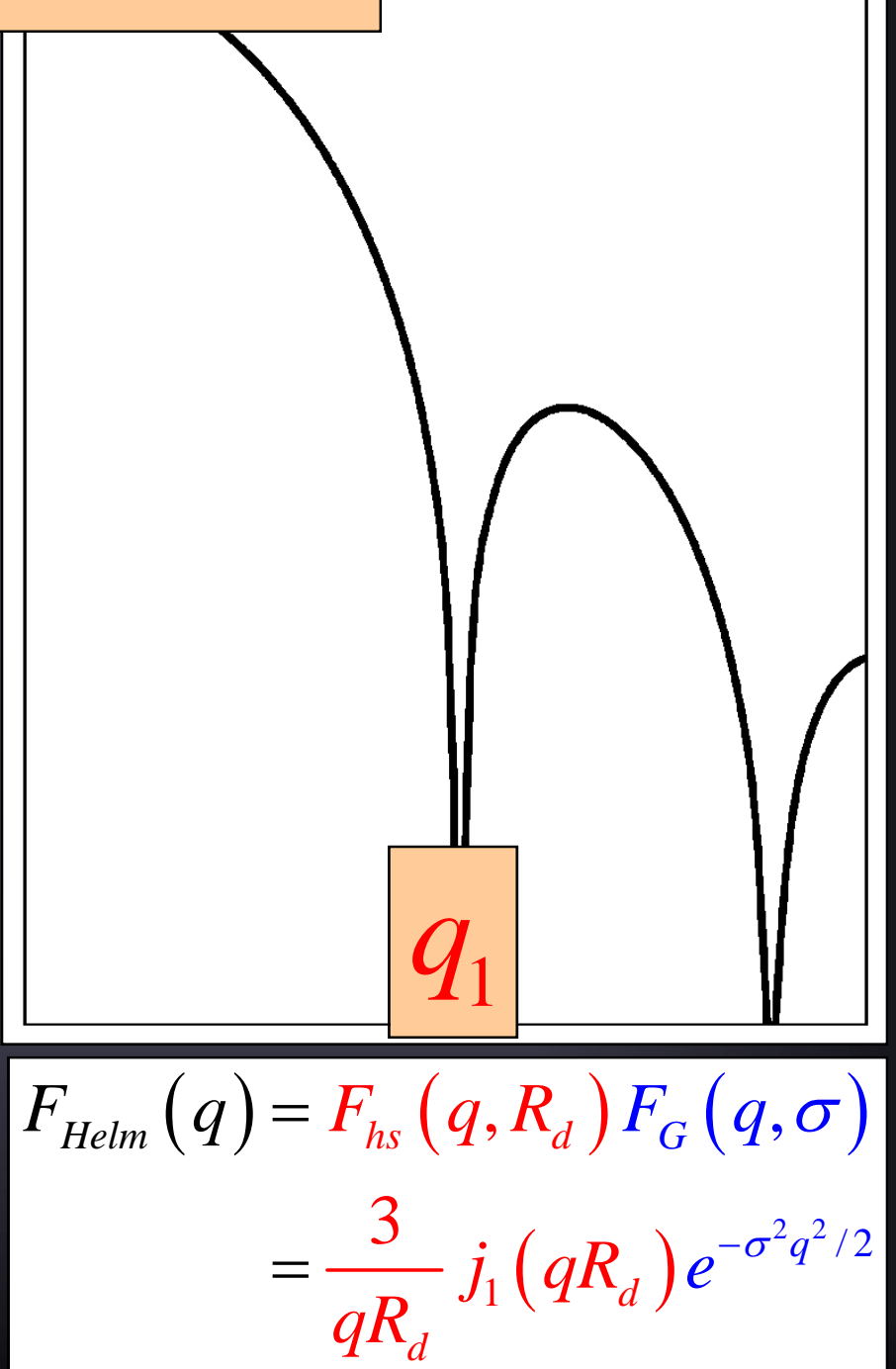




\section{Helm model}

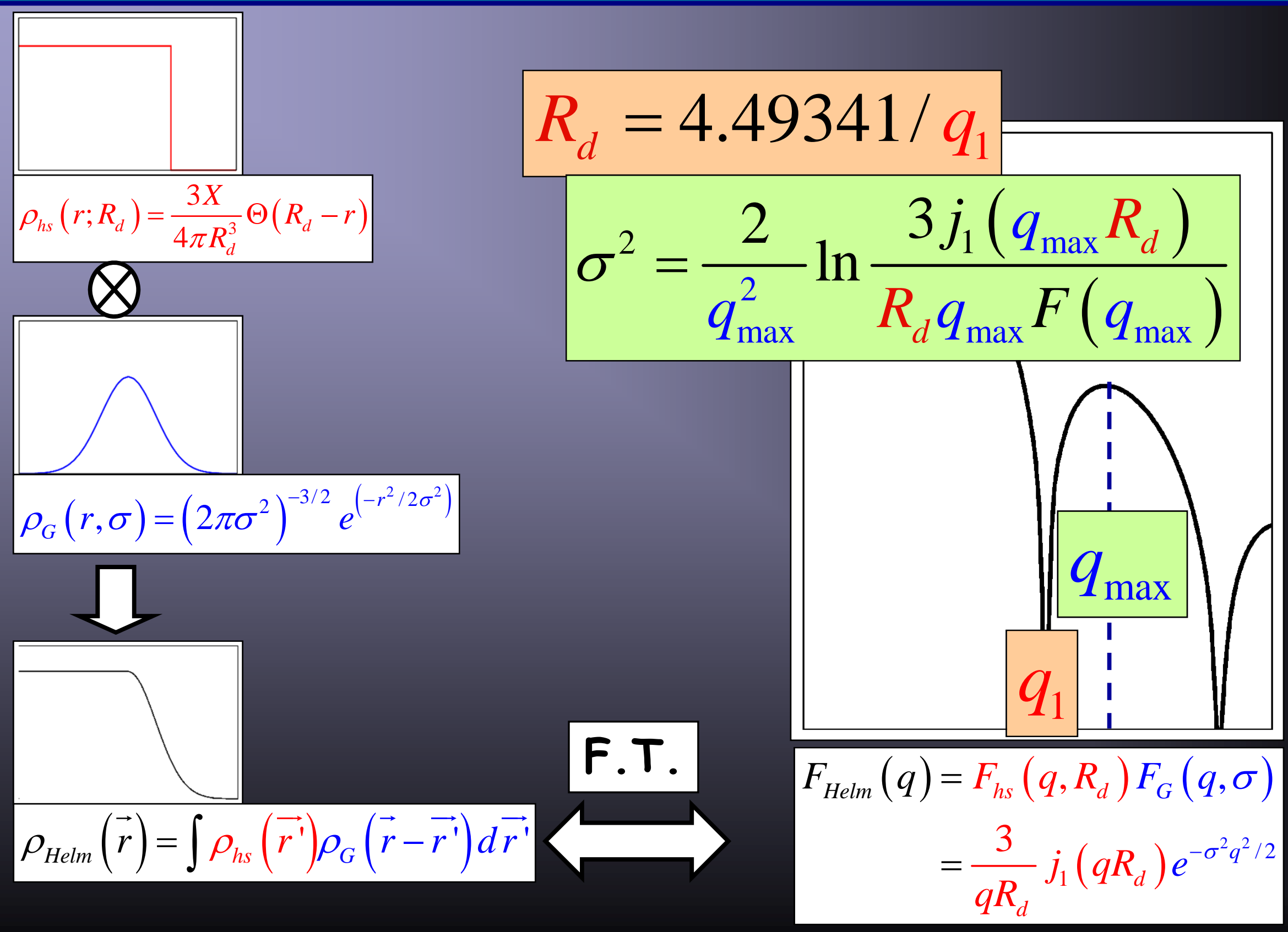




\section{Isotopic chains}

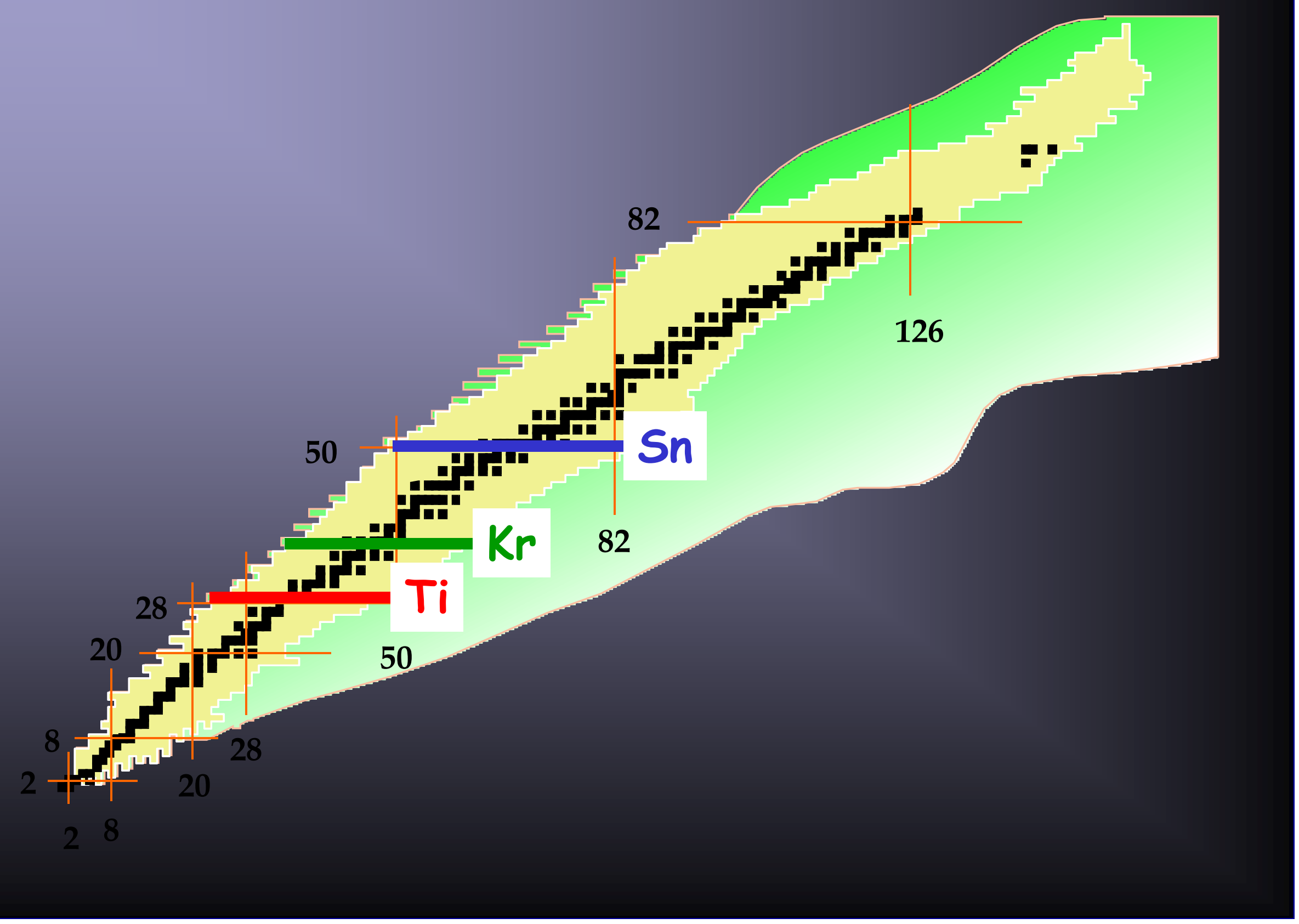




\section{Charge rms radii}

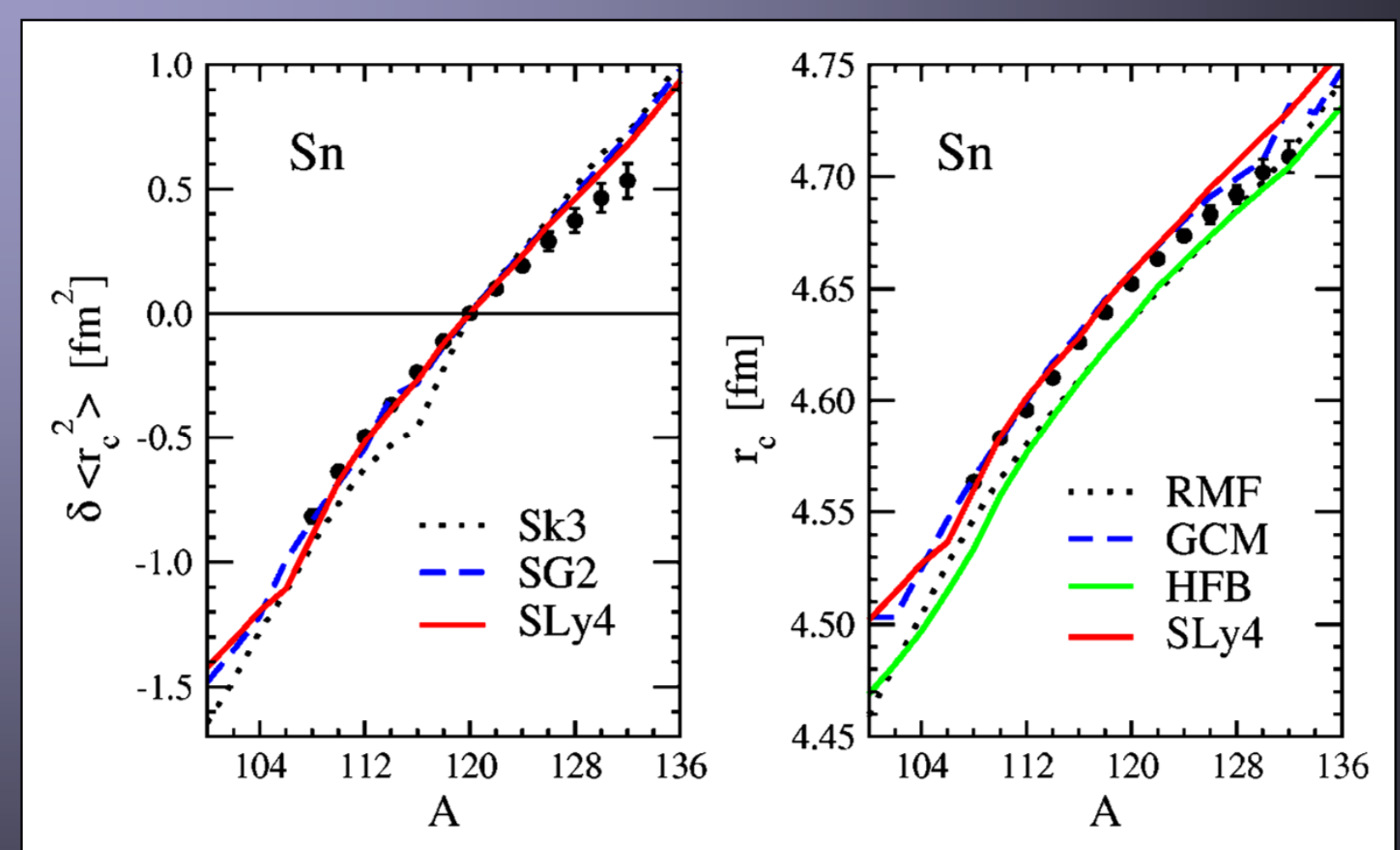

Charge rms radii of tin isotopes

$\left\langle r_{c h}^{2}\right\rangle=\left\langle r_{p}^{2}\right\rangle+\left\langle r_{c h}^{2}\right\rangle_{p}+(N / Z)\left\langle r_{c h}^{2}\right\rangle_{n}+r_{C M}^{2}+r_{S O}^{2}$ 


\section{Proton and neutron rms radii}

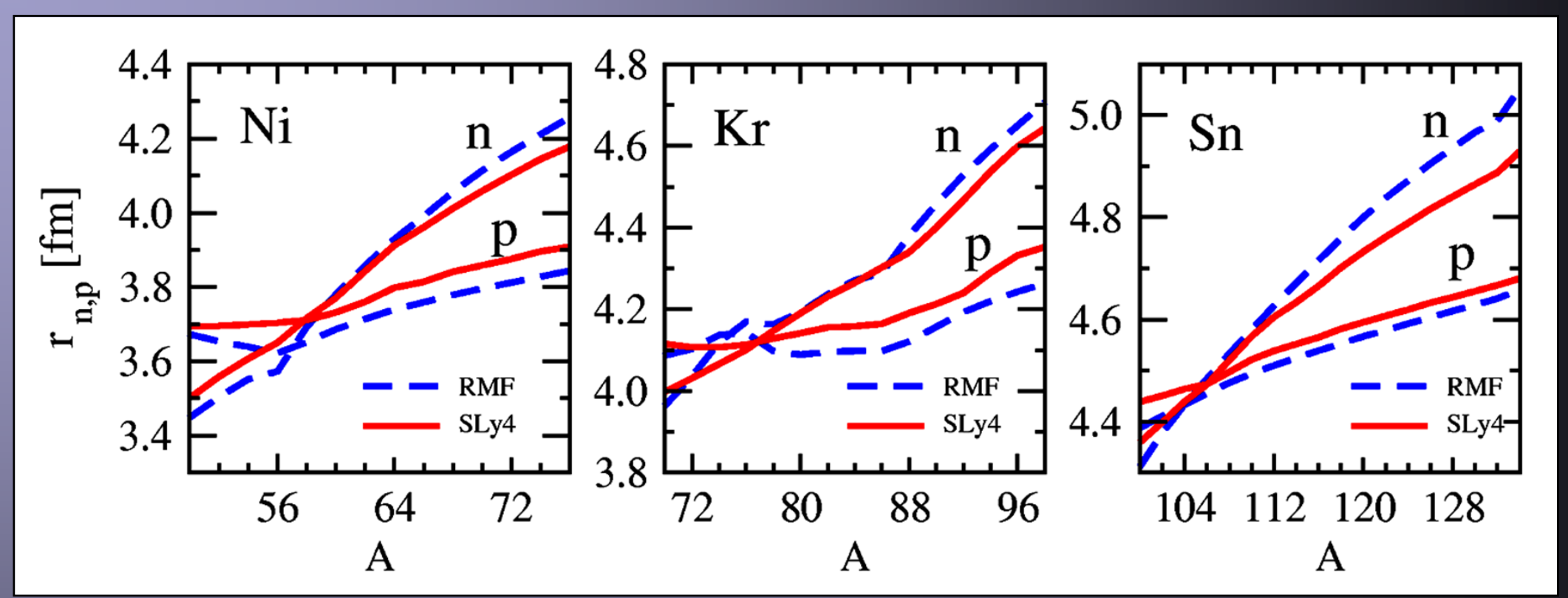

$\Delta r_{n p}=\left\langle r_{n}^{2}\right\rangle^{1 / 2}-\left\langle r_{p}^{2}\right\rangle^{1 / 2}$

Hadron scattering Antiproton atoms GDR SDR
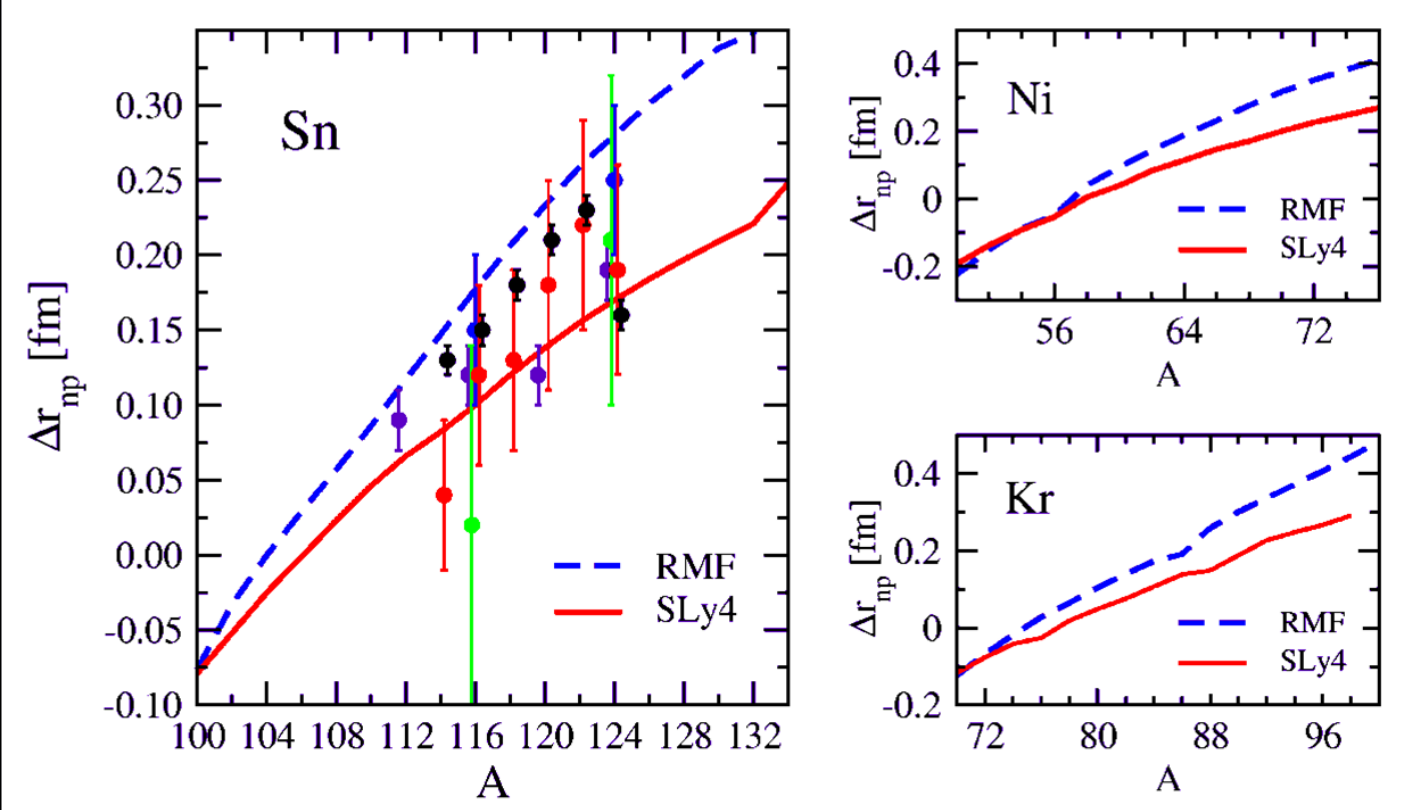


\section{Neutron-skin definitions (based on radii)}

$$
R_{h s}=\sqrt{5 / 3}\left\langle r^{2}\right\rangle^{1 / 2}
$$

$$
R_{d}=4.49341 / q_{1}
$$

$$
\Delta R_{X}=R_{X, n}-R_{X, p}
$$

$$
R_{\text {Helm }}=\sqrt{5 / 3} R_{r m s}^{\text {Helm }}=\sqrt{R_{d}^{2}+5 \sigma^{2}}
$$




\section{Neutron-skin definitions (based on density tails)}

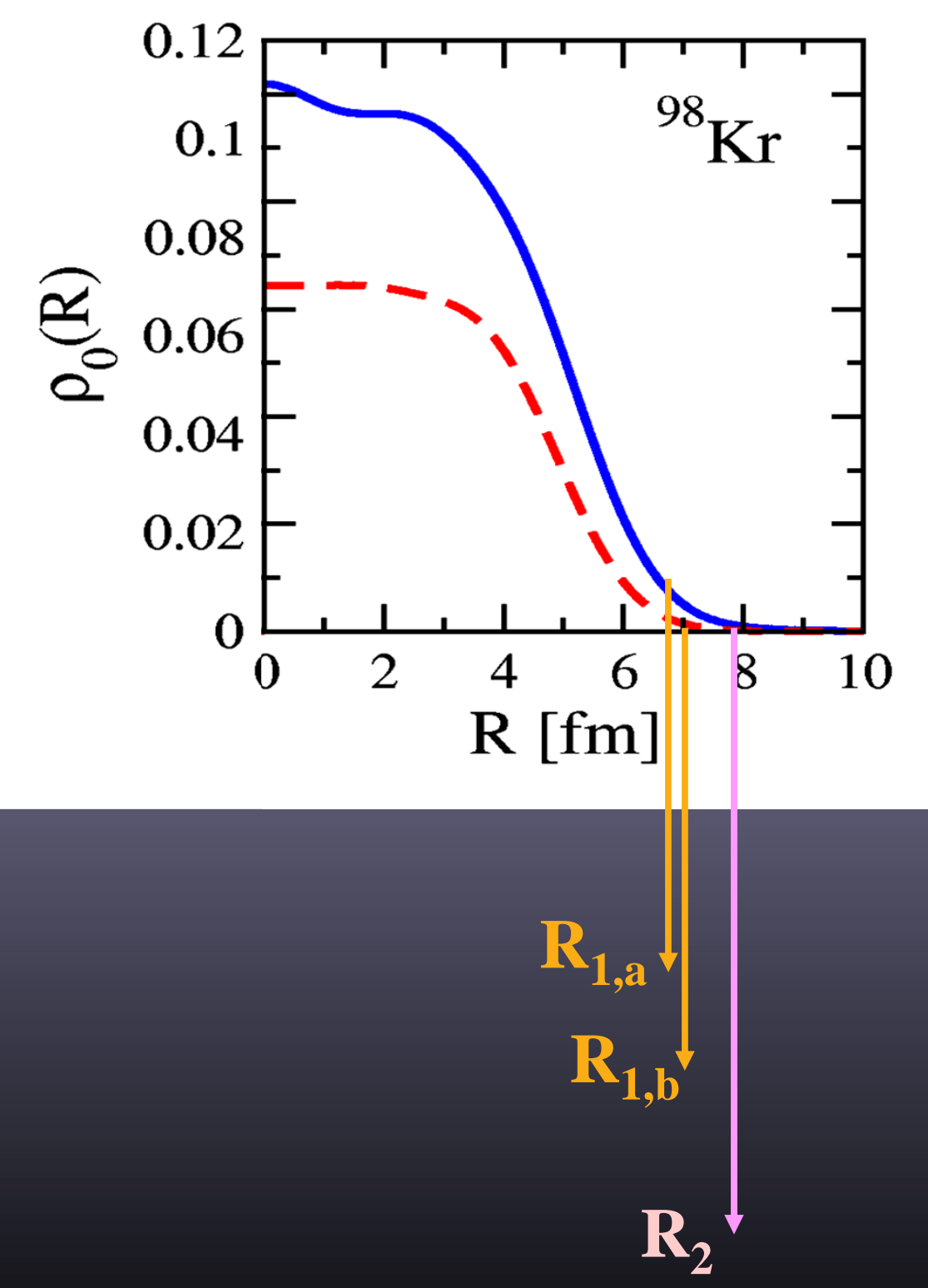

$$
\begin{array}{ll}
R_{2}: & \frac{\rho_{n}\left(R_{2}\right)}{\rho_{n}(0)}=0.01 \\
R_{1, a}: & \frac{\rho_{n}\left(R_{1, a}\right)}{\rho_{p}\left(R_{1, a}\right)}=4 \\
R_{1, b}: & \frac{\rho_{p}\left(R_{1, b}\right)}{\rho_{p}(0)}=0.01 \\
\Delta R=R_{2}-R_{1}
\end{array}
$$

Fukunishi et al. PRC48 (93) 1648 


\section{$H F+B C S$ proton and neutron densities $\rho_{0}(R)$}

\section{$\mathrm{Ni}$ isotopes}

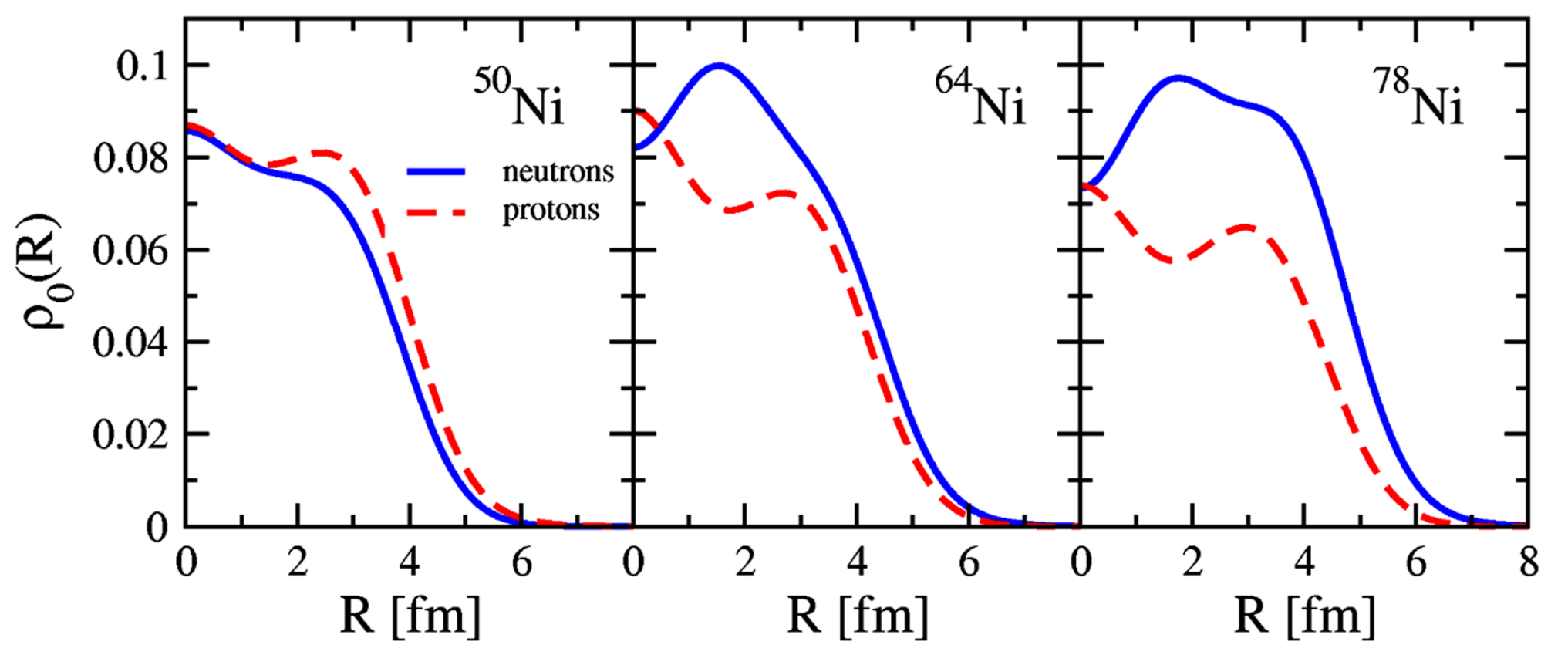




\section{$H F+B C S$ proton and neutron densities $\rho_{0}(R)$}

$\mathrm{Ni}$ isotodes

$\mathrm{Kr}$ isotopes

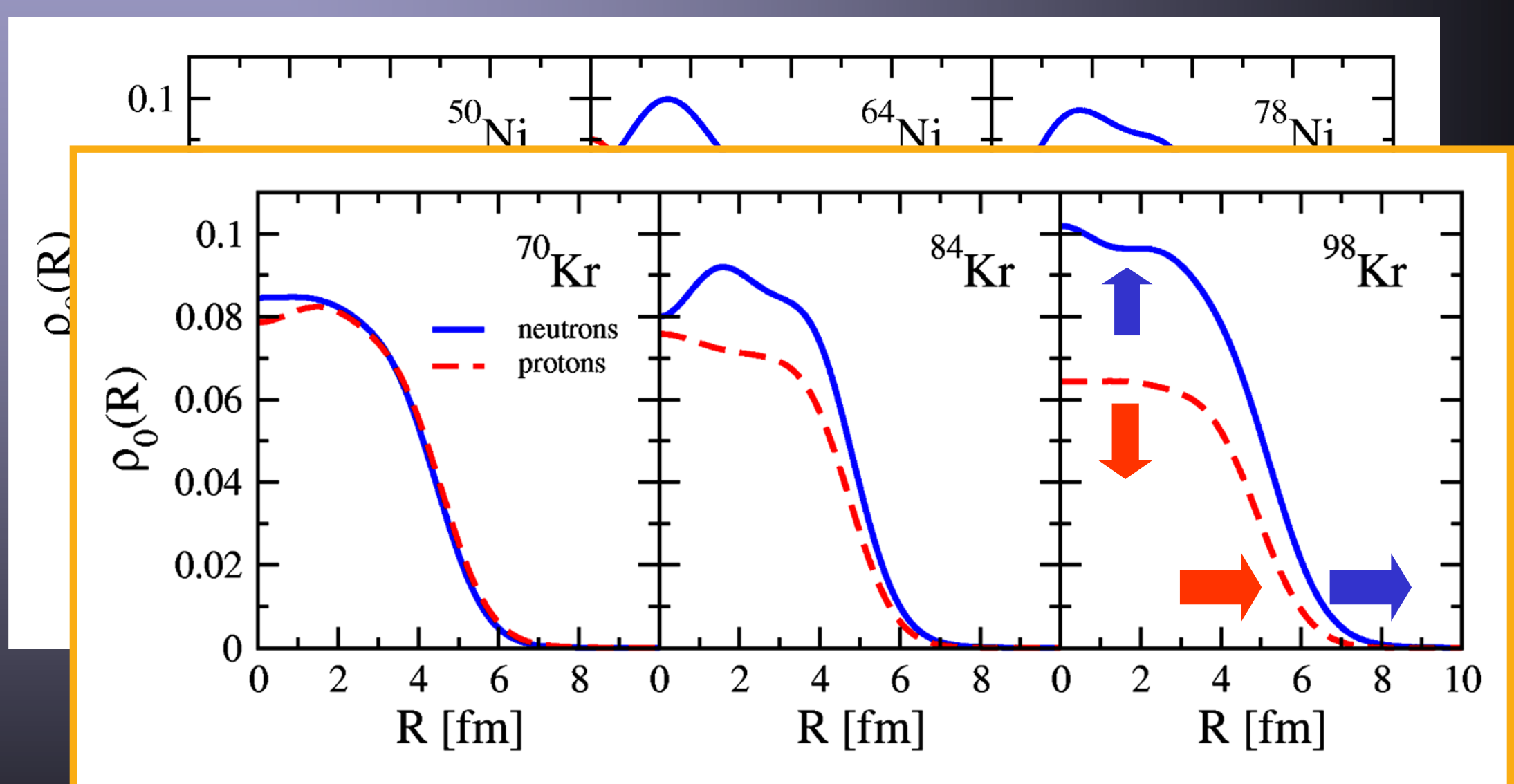




\section{$H F+B C S$ proton and neutron densities $\rho_{0}(R)$}

\section{$\mathrm{Ni}$ isotopes}

Kr ientanoe

\section{$\mathrm{Sn}$ isotopes}
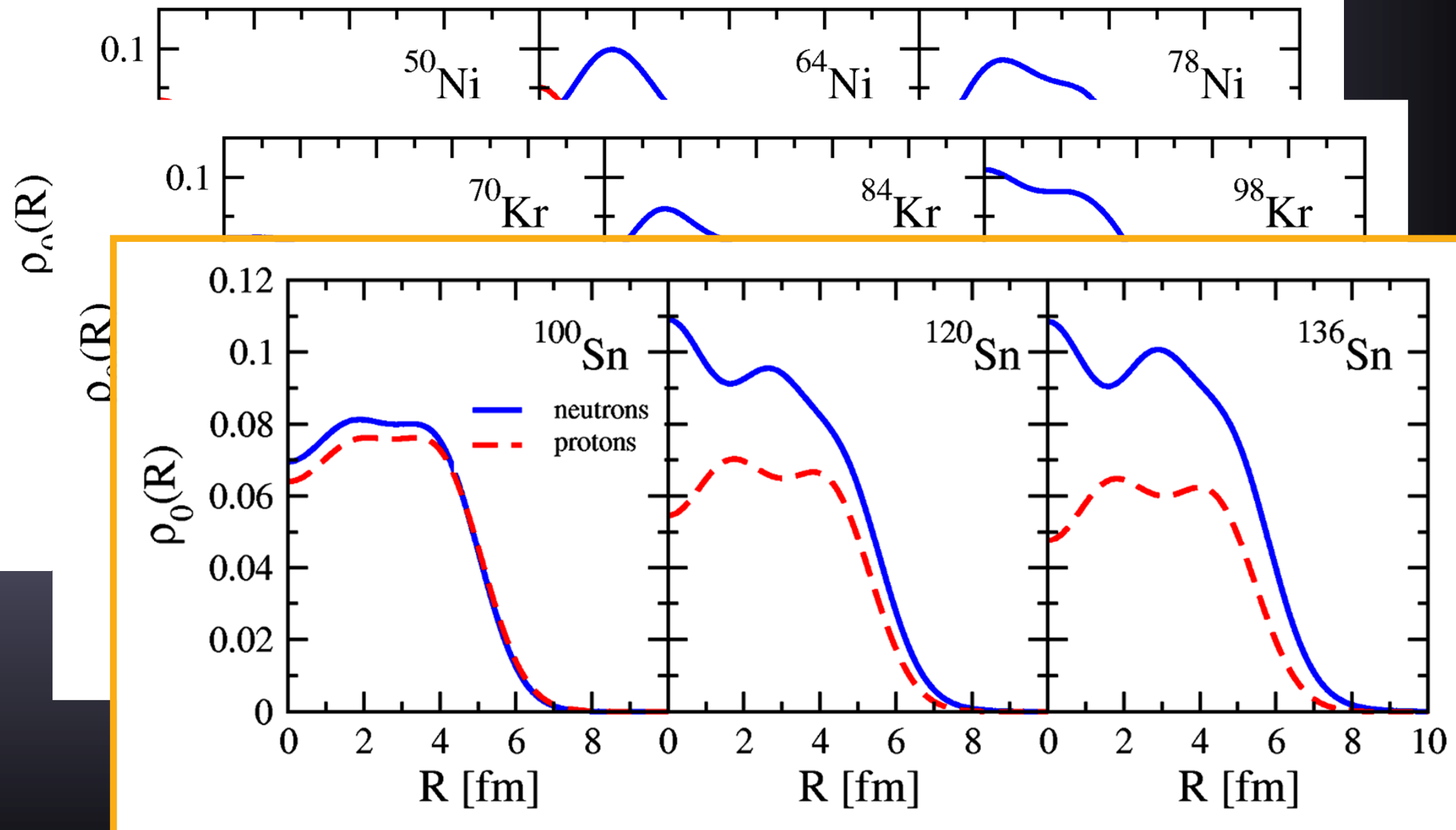


\section{Neutron skin thickness}
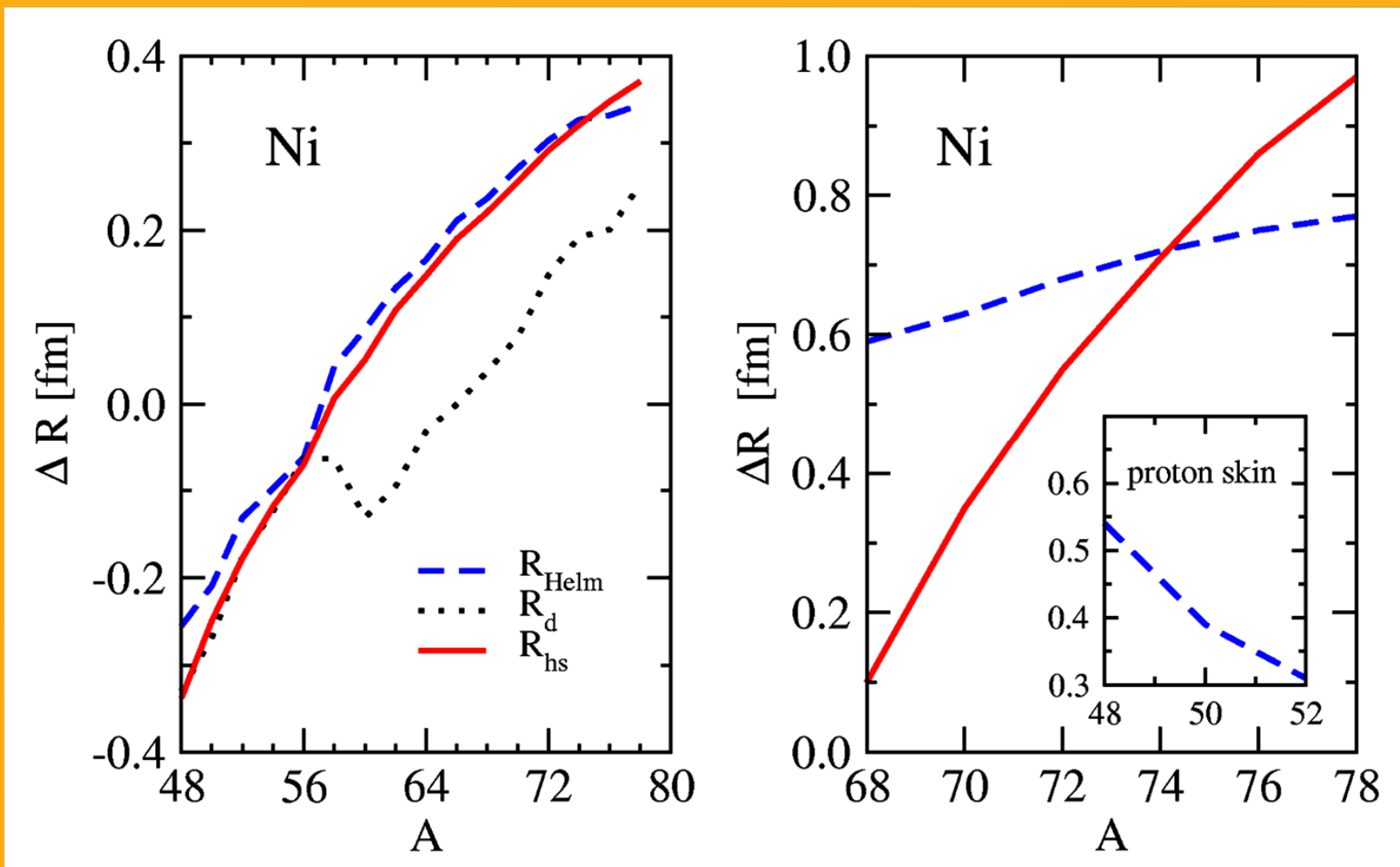

$\Delta R_{d}=R_{d}[n-p]$

$\Delta R_{\text {Helm }}=\sqrt{R_{d}^{2}+5 \sigma^{2}}[n-p]$

$\Delta R=R_{2}-R_{1, a}$

$\Delta R=R_{2}-R_{1, b}$

$\Delta R_{h \mathrm{~s}}=\sqrt{5 / 3}\left\langle r^{2}\right\rangle^{1 / 2}[n-p]$ 


\section{Neutron skin thickness}

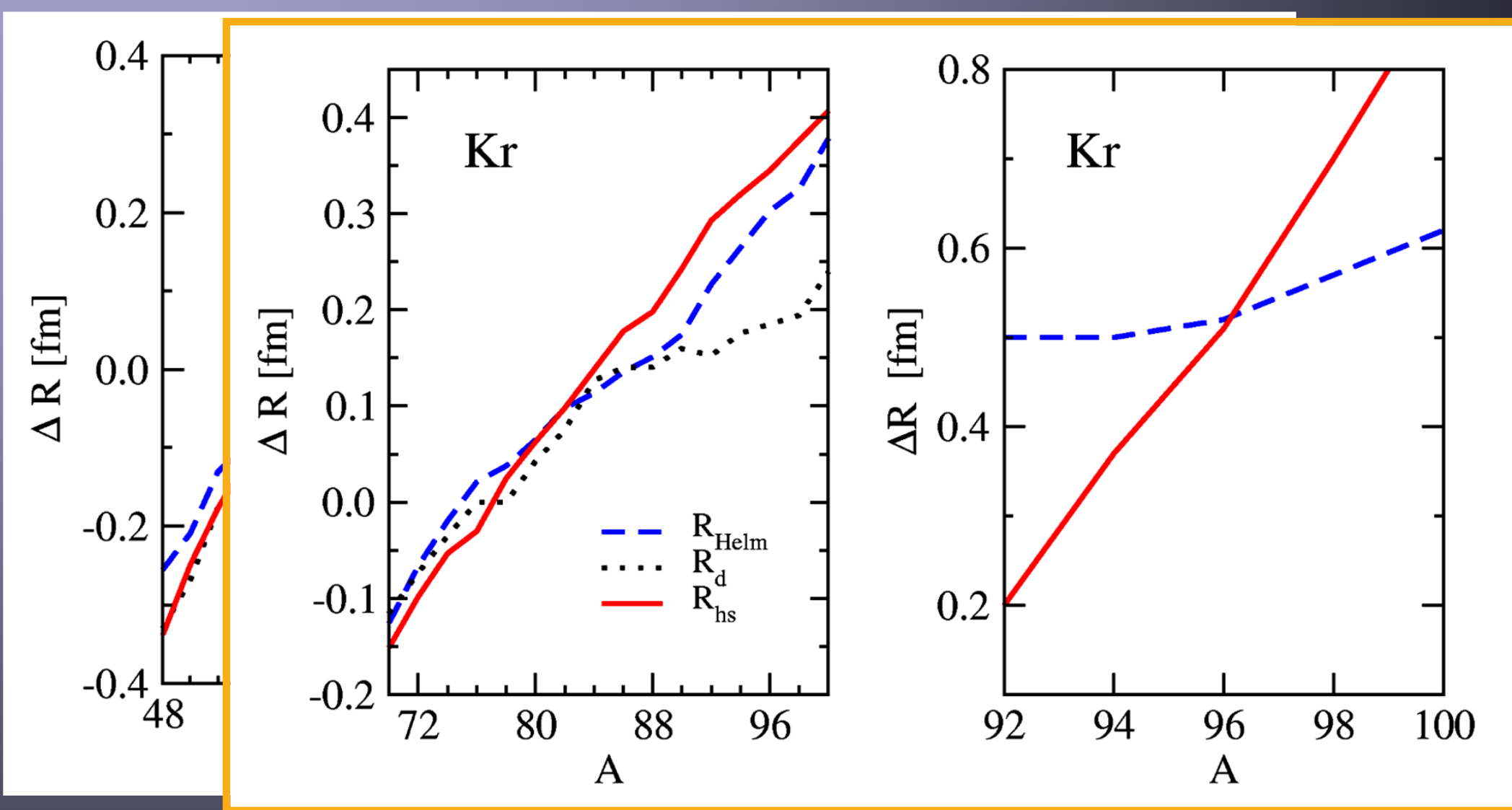

$\Delta R_{d}=R_{d}[n-p]$

$\Delta R_{\text {Helm }}=\sqrt{R_{d}^{2}+5 \sigma^{2}}[n-p]$

$\Delta R=R_{2}-R_{1, a}$

$\Delta R=R_{2}-R_{1, b}$

$\Delta R_{h \mathrm{~s}}=\sqrt{5 / 3}\left\langle r^{2}\right\rangle^{1 / 2}[n-p]$ 


\section{Neutron skin thickness}

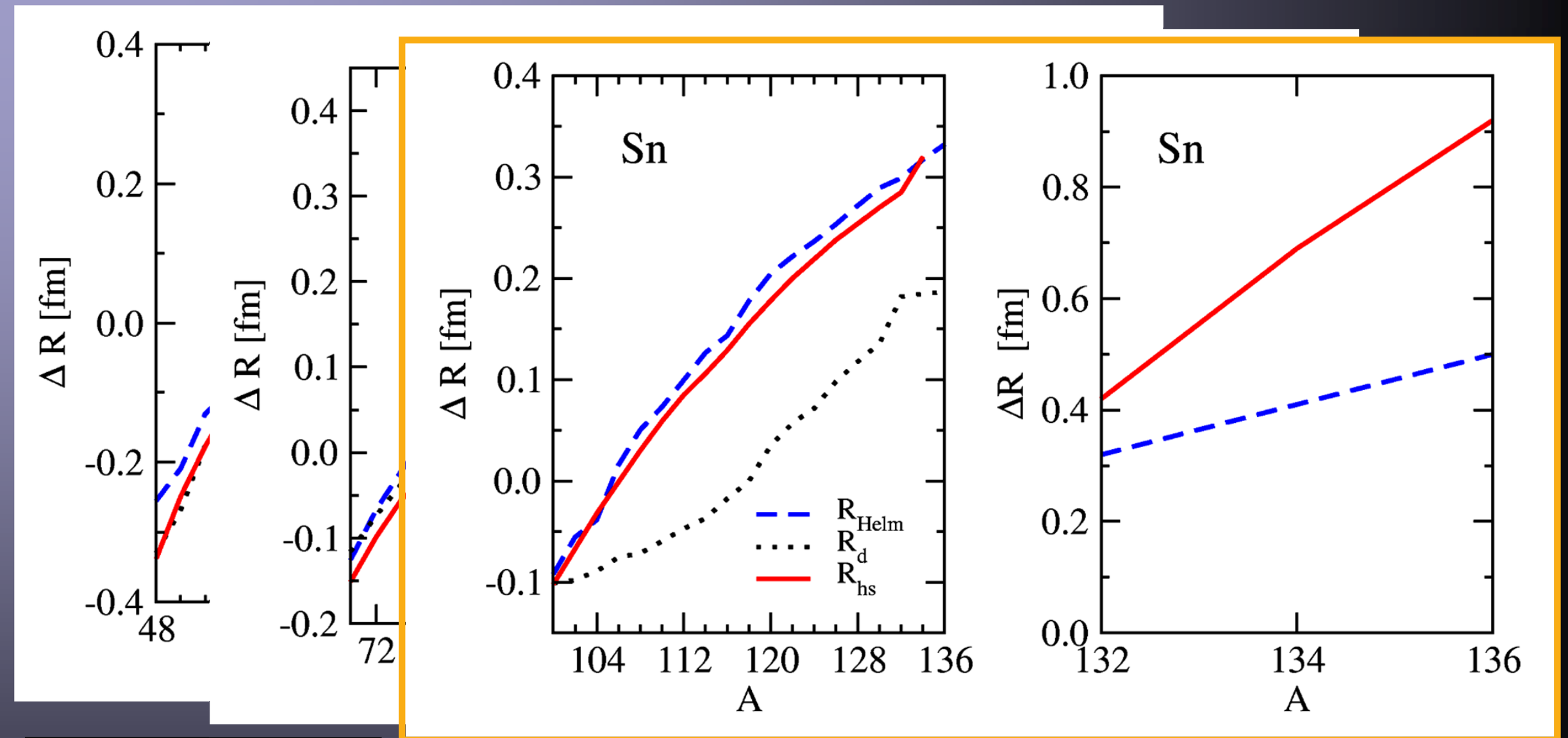

$\Delta R_{d}=R_{d}[n-p]$

$\Delta R_{\text {Helm }}=\sqrt{R_{d}^{2}+5 \sigma^{2}}[n-p]$ $\Delta R_{h \mathrm{~s}}=\sqrt{5 / 3}\left\langle r^{2}\right\rangle^{1 / 2}[n-p]$

$$
\begin{aligned}
& \Delta R=R_{2}-R_{1, a} \\
& \Delta R=R_{2}-R_{1, b}
\end{aligned}
$$




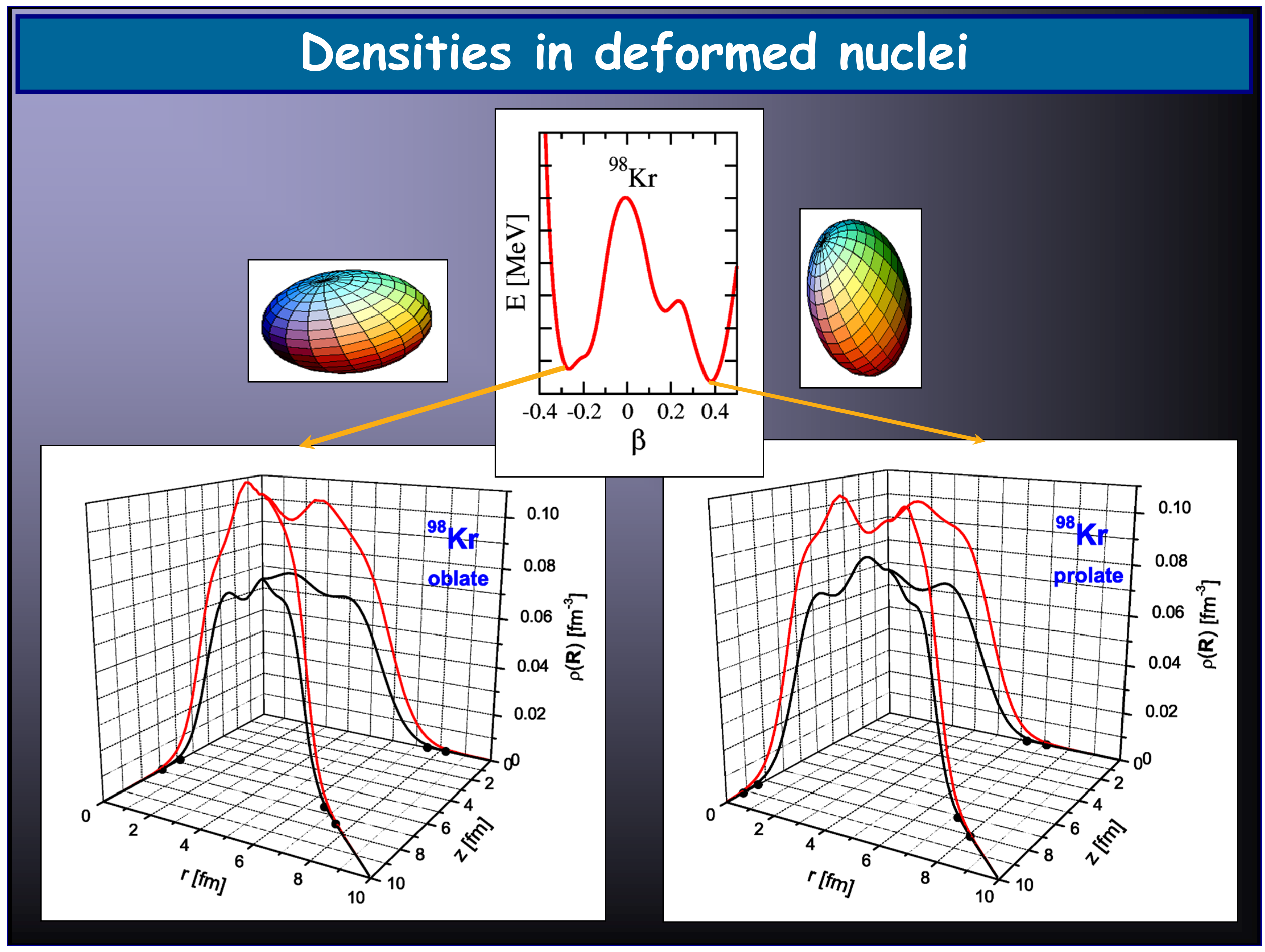




\section{Densities in deformed nuclei}

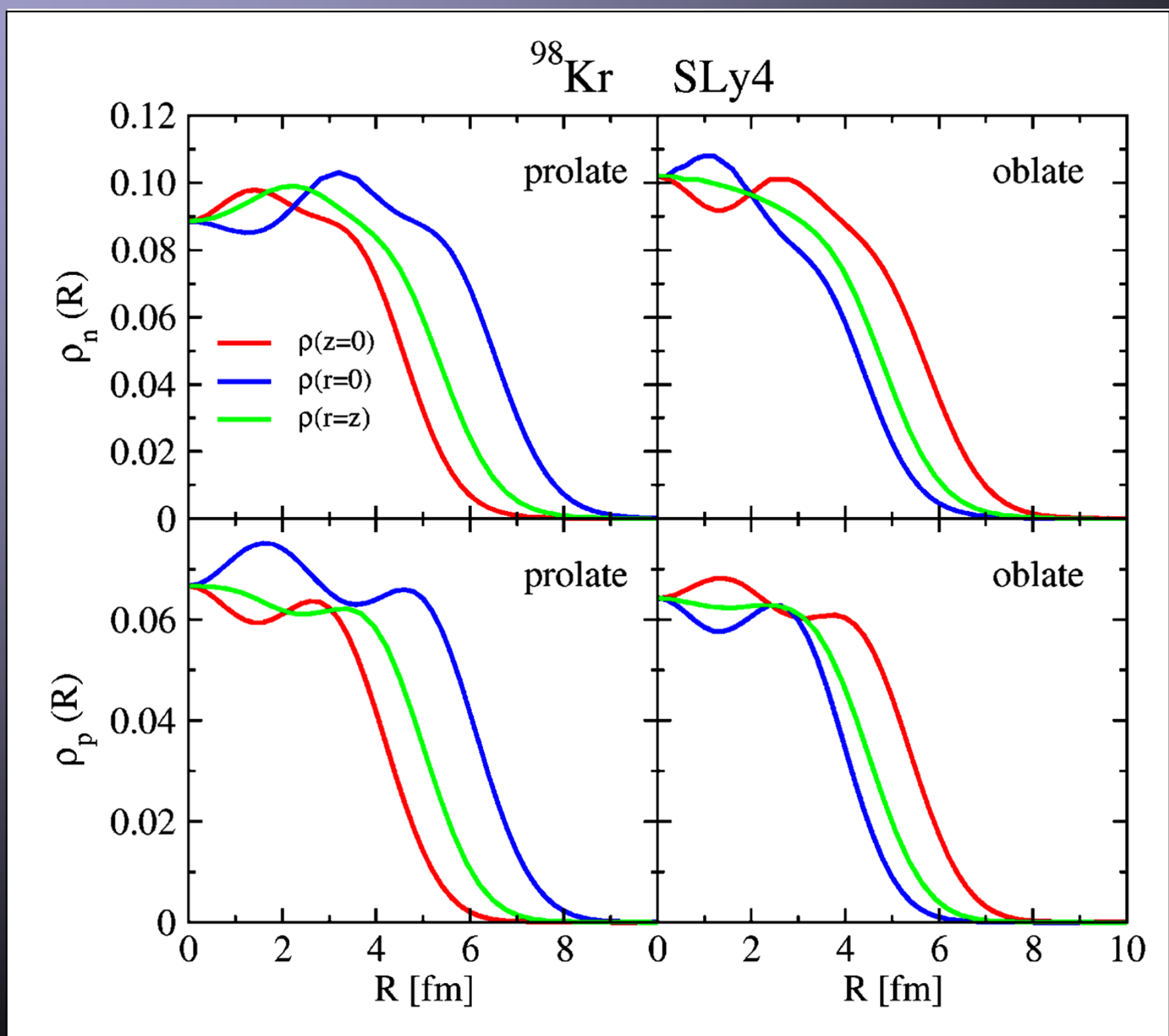




\section{Densities in deformed nuclei}

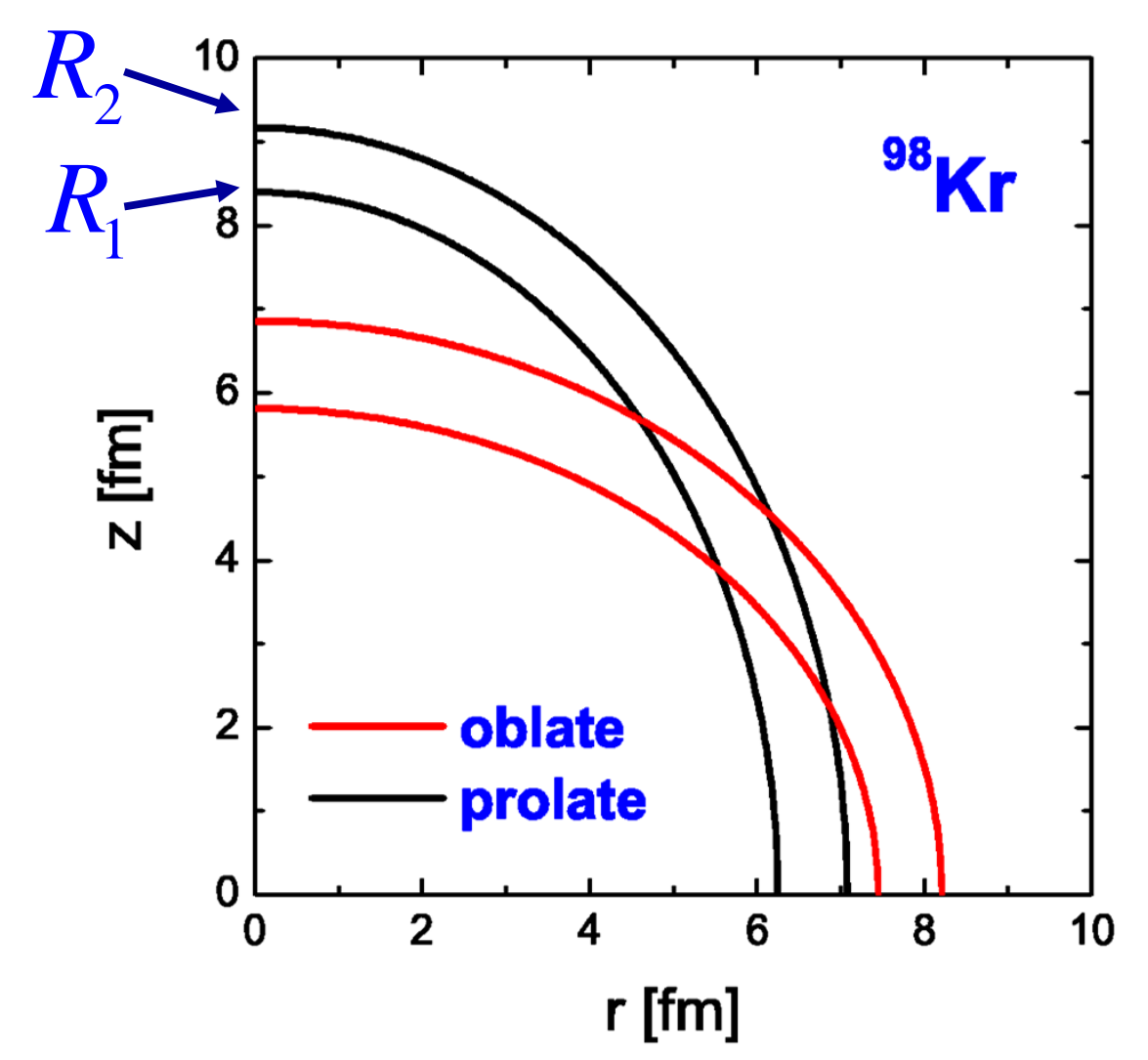




\section{Conclusions}

Theoretical approach based on deformed Skyrme (SLy4) HF+BCS

- Three isotopic chains (Ni, Kr, Sn) chains

- Good agreement with experiment (charge radii)

- Calculate proton and neutron density distributions (and radii)

Explore

- Different definitions of neutron-skin thickness

- Skin formation in isotopic chains

- Neutron-skin along different directions in deformed nuclei

Find

- Strong dependence of the neutron-skin thickness on the various definitions. Linear increase with $\mathrm{N}$

-While the radial extensions of the densities in deformed nuclei (prolate and oblate) change with the direction, the neutron-skin thickness remains practically constant along the different directions 


\section{Multipolar densities in deformed nuclei}

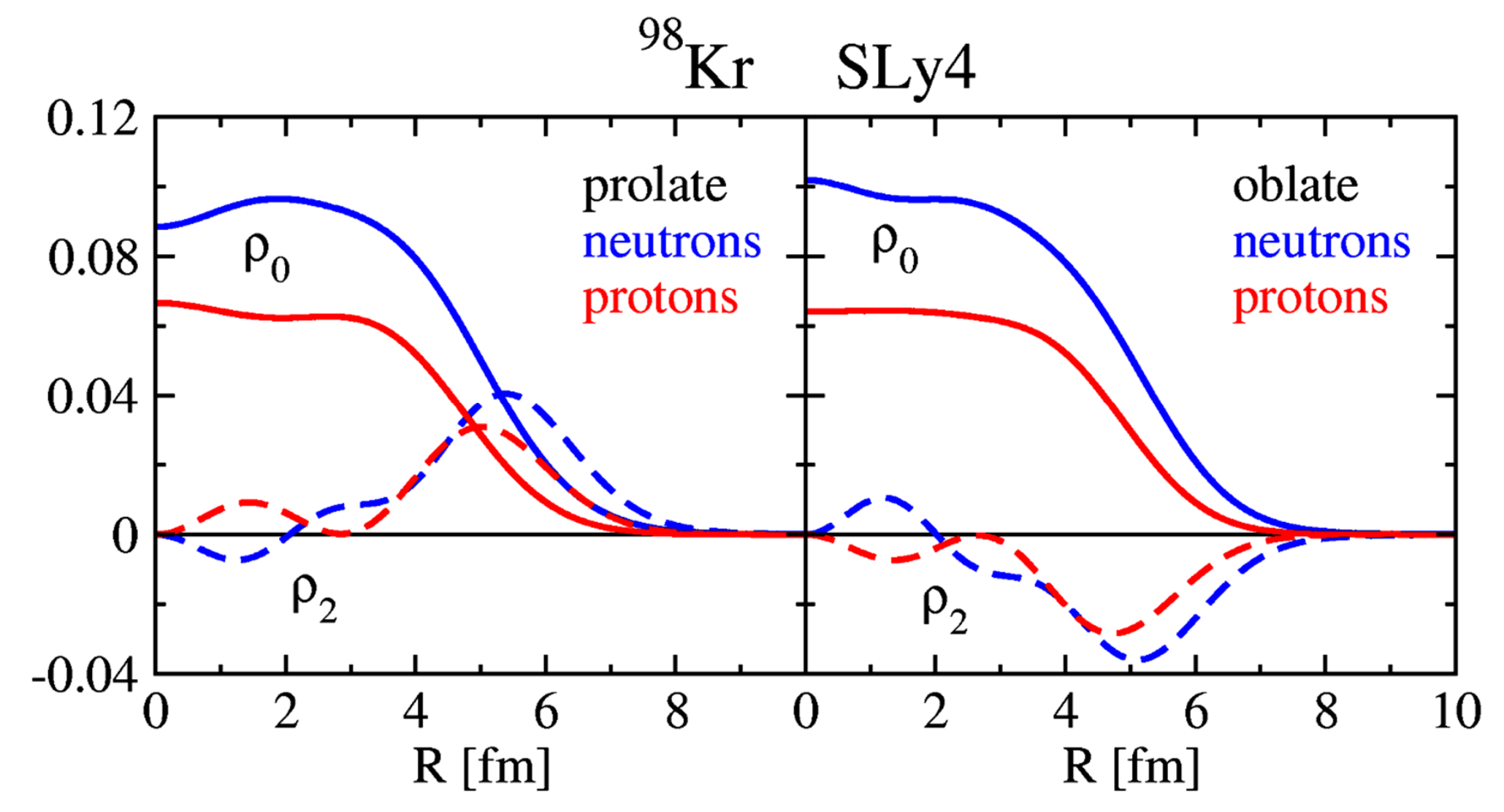

$$
\rho(r, z)=\sum_{\lambda} \rho_{\lambda}(R) P_{\lambda}(\cos \theta)=\rho_{0}(R)+\rho_{2}(R) P_{2}(\cos \theta)+\cdots
$$




\section{Appendix}
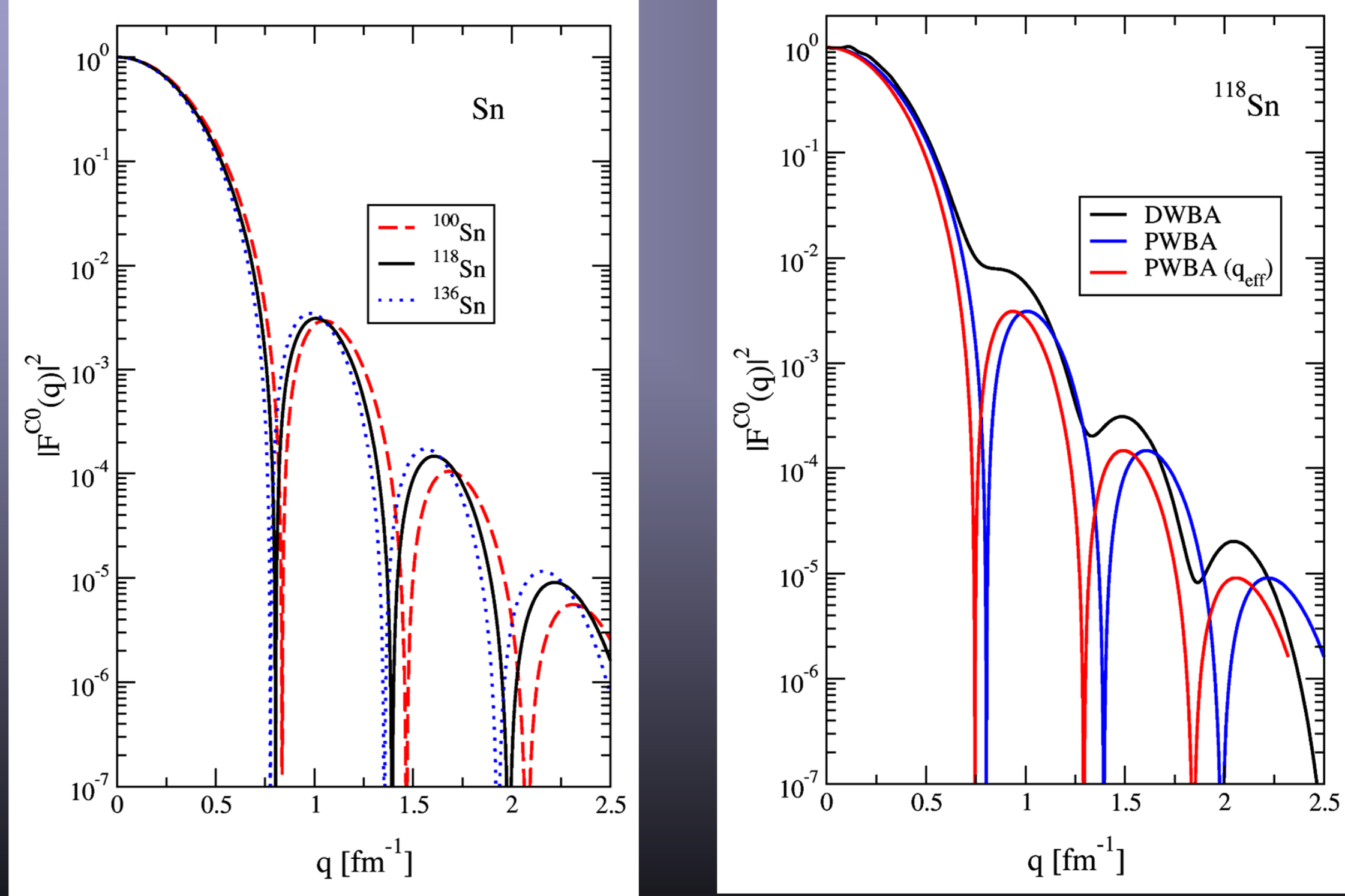